\title{
La problématique de la mise en œuvre du principe d'égalité en droit burundais de la famille. Cas des droits successoraux de la femme.
}

\author{
Par Alexis Manirakiza ${ }^{l}$
}

"L'avenir de l'homme est la femme " (Louis Aragon, Le Fou d'Elsa). "Il est plus facile de proclamer l'égalité que de la réaliser(Edouard Herriot, Aux sources de la liberté. ").

\section{INTRODUCTION}

Le principed'égalitéforme avec son corollaire qu'est la non- discriminationdes 'piliers jumeaux'sur lesquelsrepose tout l'édifice des droits humains. ${ }^{2}$ Ces principessont en plus considérés comme des véritables droits autonomes. ${ }^{3}$ Ainsi, à l'échelle internationale, régionale et même locale, beaucoup de textes reconnaissant et proclamantces droitset ces principes existent.Avec l'internationalisation de l'Etat de droit, la plupart des Etats les ont ratifiés. Le Burundi n'est pas en reste. Il aen effet souscrit à pas mal de textes internationaux consacrant l'égalité etprohibant la discrimination.Il s'est même doté d'une constitution que l'on peut qualifier de très moderne si l'on passe au peigne fin ses dispositionsen ce qui concerne les principes d'égalité et denon-discrimination.

Et pourtant, la proclamation des droits ne suit pas forcément leur mise enœuvre effective. Un écart s'observe entre les principes proclaméset la réalité quotidienne.

Pour s'en rendre compte, cet articleévoquela problématique de la mise en œuvre du principe d'égalité en droit burundais de la famille, plus particulièrement en ce qui concerne les droits successoraux des femmes.

En effet, force est de constater que malgré la consécration du principe d'égalité et de non-discrimination en droit burundais, les règles de dévolution successorale restent toujours empreintes d'une certaine idée de discrimination à l'égard des femmes. Cela se remarquedavantagedans le milieu rural que dans le milieu urbain.

1 Master en Droit de l'Université catholique de Louvain(Belgique), Candidat Doctorant à l'Université d'Anvers(Belgique), Maître-Assistant à la Faculté de Droit de l'Université du Burundi, Courriel : allyman100@gmail.com ou alexis.manirakiza@uantwerpen.be

2 Manisuli Ssenyonjo (2007). Culture and the Human Rights of Women in Africa: Between Light and Shadow, Journal of African Law, 51, ${ }^{\circ} 1$, p.42, voir aussiManisuli Ssenyonjo, Women's Rights To Equality And Non-Discrimination: Discriminatory Family Legislation In Uganda And The Role Of Uganda's Constitutional Court, International Journal of Law, Policy and the Family21 (2007), p.341.

3 Le titre même de ce dernier article est évocateur sur l'existence du droit à l'égalité et à la non-discrimination. 
La question qui se pose estcelle de savoir si cet état de fait est acceptable et si les juges ne devraient pas s'affranchir de ces règles discriminatoires en appliquant des textes qui sonten phase avec l'égalité.

L'article est divisé en trois principales parties.

La première partie de l'article estdédiéeà l'analyse de la consécration normative de l'égalité et la non-discrimination en droit burundais.

Dans la deuxième partie, il sera fait observerla façon dont ces principes sont mis en œuvre dans le domaine successoral. Il sera démontré que, malgré l'évolution du droit coutumier en la matière, cette mise en œuvre est loin d'être effective,surtout enmilieu rural, alors qu'elle est de plus en plus acceptée dans le milieu urbain. C'est ce que nous appellerons un dualisme jurisprudentiel. Les raisons qui expliquent cet état de faitseront analysées ainsi que leur non pertinence.

Dans la troisième partie, il sera discuté des solutionsque le juge devraitretenir en cas de conflits successoraux impliquant les femmes et qui sont en concordance avec le principe d'égalité.

\section{LE PRINCIPE D'EGALITE ET DE NON-DISCRIMINATION EN DROIT BURUNDAIS : UNE CONSECRATIONSANS RESERVE}

S'il y a un crédit qu'il faut reconnaître au législateur burundais, c'est de consacrer et affirmer dans les textesla plupart des principes fondamentaux des droits de la personne humaine. L'égalité et la non discrimination vont dans ce sens et sont reconnussans réserve par le législateur burundais. Il aen effetratifiéla plupart des principaux instruments internationaux proclamant l'égalité et prohibant la discrimination(A). Bien plus, il s'est doté d'une constitution qui va également dans le sens de prohiber la discrimination et de promouvoir l'égalité(B).

Pour des besoins pratiques, cet article se limitera au développement de quelques instruments internationaux jugés importants et qui sont dans un lien étroit avec l'égalité hommefemme. 


\section{A. QUELQUESTEXTES INTERNATIONAUX RECONNAISSANT L'EGALITEET LA NON-DISCRIMINATIONLIANT LE BURUNDI}

1. Déclaration universelle des droits de l'homme

Parmi les textes fondateurs de proclamation et de protection des droits de l'homme, la DUDH $^{4}$ est considéré comme le plus important.Elle est on ne peut plus clair sur le principe d'égalité et de non-discrimination ${ }^{5}$.

Comme unedéclaration n'est pas susceptible d'être ratifiée,et donc n'est pas par sa nature contraignante ${ }^{6}$, il peut se poser la question de savoir dans quelle mesurela DUDH oules droits et garanties qu'elle proclame lie(nt) le Burundi. Les arguments qui suiventaident à répondre à la question et démontrent quele Burundi est lié par la DUDH.

Premièrement, l'article 19 de la Constitution de la République du Burundi a donné aux droits et garanties proclamés par la Déclarationune nature constitutionnelle ${ }^{7}$ en ces termes :

"Les droits et devoirs proclamés et garantis, entre autres, par la Déclaration universelle des droits de l'homme (....) font partie intégrante de la Constitution de la République du Burundi. »

Deuxièmement, la Cour constitutionnelle du Burundia déjà invoquédans certains de ses arrêts, certains articles de la DUDH. Comme l'a fait remarquer StefVandenginste, la Cour Constitutionnelle a confirmé que le Burundi est lié par la DUDH et qu'en même temps le Burundi, dans la domestication du droit international, connait le système moniste. Nous y reviendrons.

4 Elle a été adoptéeet proclaméeen 1948 par l'Assemblée générale des Nations Unies dans sa Résolution 217 A(III) du 10 décembre 1948. Voir Codes et lois du Burundi (31 déc.2006), tome I, p.151.

5 D'abord, selon son article 1, "Tous les êtres humains naissent libres et égaux en dignité et en droits. Ils sont doués de raison et de conscience et doivent agir les uns envers les autres dans un esprit de fraternité. ». Elle continue dans son article 2.1, "Chacun peut se prévaloir de tous les droits et de toutes les libertés proclamés dans la présente Déclaration, sans distinction aucune, notamment de race, de couleur, de sexe, de langue, de religion, d'opinion politique ou de toute autre opinion, d'origine nationale ou sociale, de fortune, de naissance ou de toute autre situation ».Selon aussil'article 7 « Tous sont égaux devant la loi et ont droit sans distinction à une égale protection de la loi. Tous ont droit à une protection égale contre toute discrimination qui violerait la présente Déclaration et contre toute provocation à une telle discrimination ». Selon l'article 16, «A partir de l'âge nubile, l'homme et la femme, sans aucune restriction quant à la race, la nationalité ou la religion, ont le droit de se marier et de fonder une famille. Ils ont des droits égaux au regard du mariage, durant le mariage et lors de sa dissolution ".

6 Sur la nature juridique de la déclaration, voir Vandenginste, S. (2011), Stones Left Unturned: Law as a Source and Instrument of Transitional Justice in Burundi. Antwerp, Intersentia, p.285 qui réfère à H. Hannum "The Status of the Universal Declaration of Human Rights in National and International Law, Georgia Journal of International and Comparative Law, Vol 25,1995-1996,289-394.

7 Voir LOI $\mathrm{n}^{\circ} 1 / 610$ du 18 mars 2005 portantpromulgation de la Constitution de la République du Burundi. (B.O.B., 2005, nº 3ter, p. 1.). 


\section{Le pacte international relatif aux droits civils et politiques ${ }^{8}$}

Contrairement à la DUDH dont le caractère contraignant n'apparaît pas d'emblée, le pacte international relatif aux droits civils et politiquesa lui une nature contraignante et lie le $\mathrm{Bu}$ rundi.

D'abord, contrairement à la DUDH qui ne nécessitait pas une ratification pour sa mise en vigueur, le PIDCP peut être ratifié. Et le Burundi y a procédé par une loi n ${ }^{\circ}$ 1/009 du 14 mars $1990^{9}$.

Ensuite, la Constitution burundaise, au travers de son article 19, a donné une nature constitutionnelle aux droits et devoirs proclamés et garantis par le Pacte en ces termes

"Les droits et devoirs proclamés et garantis, entre autres (....) les Pactes internationaux relatifs aux droits de l'homme (....) font partie intégrante de la Constitution de la République du Burundi.»

Bien plus, comme cela a été dit à propos de la DUDH, la Cour Constitutionnelle du Burundi, a dans ses nombreux arrêts, déclaré que desarticlesdu PIDCP sontapplicablesau Burundi. ${ }^{10}$. Nous y reviendrons.

3. Le pacte international relatif aux droits économiques, sociaux et culturels ${ }^{11}$

L'égalité et la non discrimination s'appliquent aussi en ce qui est des droits économiques, sociaux et culturels ${ }^{12}$.

8 Adopté et ouvert à la signature, à la ratification et à l'adhésion par l'Assemblée générale des Nations Unies dans sa résolution 2200 A (XXI) du 16décembre 1966 (New York);le PIDCP est entréen vigueur le 23mars 1976.Voir Codes et lois du Burundi (31 déc.2006), tomeI,p.156.

9 Voir Codes et Lois du Burundi, T1, p.156.

10 Voir les arrêts RCCB 8 du 31 mars 1993, RCCB 18 du 8 novembre 1993, RCCB 54 du 5 octobre 1995. Et parmi les articles du PIDCP figurent en bonne partie les articles reconnaissant l'égalité. En effet, l'article 2.1 dispose « Les États parties au présent Pacte s'engagent à respecter et à garantir à tous les individus se trouvant sur leur territoire et relevant de leur compétence les droits reconnus dans le présent Pacte, sans distinction aucune, notamment de race, de couleur, de sexe, de langue, de religion, d'opinion politique ou de toute autre opinion, d'origine nationale ou sociale, de fortune, de naissance ou de toute autre situation. "L'article 26 quant à lui «Toutes les personnes sont égalesdevant la loi et ont droit sans discrimination à une égale protection de la loi. A cet égard, la loi doit interdire toute discrimination et garantir à toutes les personnes une protection égale et efficace contre toute discrimination, notamment de race, de couleur, de sexe, de langue, de religion, d'opinion politique et de toute autre opinion, d'origine nationale ou sociale, de fortune, de naissance ou de toute autre situation ». L'article 3 continue « Les Etats parties au présent Pacte s'engagent à assurer le droit égal qu'ont l'homme et la femme au bénéfice de tous les droits éco- nomiques, sociaux et culturels qui sont énumérés dans le présent Pacte. 》.

11 Le PIDESC a été adopté à la même date que le PIDCP, mais il est entré en vigueur le 3 janvier 1976. Voir Codes et Lois du Burundi (31 décembre 2006), p.153.

12 Selon l'article 2.2 du Pacte «Les Etats parties au présent Pacte s'engagent à garantir que les droits qui y sont énoncés seront exercés sans discrimination aucune fondée sur la race, la cou- 
Ce pacte est aussi obligatoire au Burundi comme il en est le cas pour le PIDCP. Il a en effet été ratifié par le Burundi, comme c'est le cas pour le PIDCP le 14 mars 1990. Et comme c'est déjà souligné, les droits et devoirsgarantis par ce pacte font partie intégrante de la constitution du Burundi.

\section{La Convention sur l'élimination de toutes les formes de discrimination àl'égard des} femmes ${ }^{13} \cdot 14$

A côté de ces trois principaux instruments ci-haut développés et qui consacraient l'égalité et la non-discriminationde manière générale,il a été nécessaire de procéder à l'adoption d'une convention plus spécifiquesur la discrimination basée sur le sexe.

En effet, ainsi que la Convention le reconnaît, malgré les efforts déployés en vue d'éliminer toute discrimination basée sur le sexe, les femmes continuent à être victimes des discriminations $^{15}$. Une protection spécifique pour les femmes étaitalors requise. C'est ainsi que les Etats parties se sont engagésà des obligations concrètes visant à l'élimination de toute discrimination de jure et de facto ${ }^{16}$.

leur, le sexe, la langue, la religion, l'opinion politique ou toute autre opinion, l'origine nationale ou sociale, la fortune, la naissance ou toute autre situation. ".

13 Adoptée et ouverte à la signature, à la ratification et à l'adhésion par l'Assemblée générale des Nations Unies dans sa résolution 34/180 du 18 décembre 1979 (New York); la Convention est entrée en vigueur le 3 septembre 1981 conformément aux dispositions de l'article 27. Voir Codes et Lois du Burundi(31 décembre2006), p.162.

14 Pour une étude détaillée de la Convention, voir Combating Discrimination Based on Sex and Gender, in Catarina Krause and Martin Scheinin(eds.) International Protection of Human Rights(Turku :Institute for Human Rights, Abo Akademy University,2009) 205-226.

15 Voir Préambule de la Convention, paragraphe 6.

16 L'article 2 est formel «Les Etats parties condamnent la discrimination à l'égard des femmes sous toutes ses formes, conviennent de poursuivre par tous les moyens appropriés et sans retard une politique tendant à éliminer la discrimination à l'égard des femmes et, à cette fin, s'engagent à:

a. Inscrire dans leur constitution nationale ou toute autre disposition législative appropriée le principe de l'égalité des hommes et des femmes, si ce n'est déjà fait, et assurer par voie de législation ou par d'autres moyens appropriés l'application effective dudit principe;

b. Adopter des mesures législatives et d'autres mesures appropriées assorties, y compris des sanctions en cas de besoin, interdisant toute discrimination à l'égard des femmes;

c. Instaurer une protection juridictionnelle des droits des femmes sur un pied d'égalité avec les hommes et garantir, par le truchement des tribunaux nationaux compétents et d'autres institutions publiques, la protection effective des femmes contre tout acte discriminatoire;

d. S'abstenir de tout acte ou pratique discriminatoire à l'égard des femmes et faire en sorte que les autorités publiques et les institutions publiques se conforment à cette obligation;

e. Prendre toutes mesures appropriées pour éliminer la discrimination pratiquée à l'égard des femmes par une personne, une organisation ou une entreprise quelconque;

f. Prendre toutes les mesures appropriées, y compris des dispositions législatives, pour modifier ou abroger toute loi, disposition réglementaire, coutume ou pratique qui constitue une discrimination à l'égard des femmes; 
Le Burundi est l'un de ces Etats parties ${ }^{17}$.

5. La Charte africaine des droits de l'homme et des peuples ${ }^{18}$

La proclamation et la promotion des droits de l'homme ne sont pas confinées uniquement à l'échelle internationale. Des blocs régionaux se sont également dotés des conventions de promotion des droits de l'homme ${ }^{19}$. C'est le cas de l'Afrique avec la CADHP. Cette dernière reconnaît également l'importance de l'égalité et de la non-discrimination. ${ }^{20}$

La CADHP fait partie du droit burundais. En effet, outre que le Burundi l'a ratifiée ${ }^{21}$, l'article 19 de la Constitution du Burundi déjà cité donne «aux droits et garanties proclamés parla $C A D H P$ » une valeur constitutionnelle.

\section{B. LA CONSTITUTION DE LA REPUBLIQUE DU BURUNDI}

Outre que le Burundi a, comme il vient d'être démontré, consacré le principe d'égalité et de la non-discrimination dans son système juridique en ratifiant certains instruments internationaux, il a en plus constitutionnalisé ces principes.

g. Abroger toutes les dispositions pénales qui constituent une discrimination à l'égard des femmes.

17 Outre qu'il l'a ratifiéesans réserve par le D.L n 1/006 du 4 avril 1991(voir Codes et Lois, op.cit, p.162),le Burundi a reconnu, au travers de l'article 19 de la constitution, que les droits etdevoirs garantispar la Convention font partie intégrante de la Constitution.

18 . Adoptée le 27 juin 1981à Nairobi (Kenya) par le 18ème Conférence de l'Organisation de l'Unité africaine;la Charte est entrée en vigueur le 21 octobre 1986 conformément à l'article 63;.Voir Codes et Lois du Burundi(31 décembre 2006), Tome 1, p.173. Cette charte a été complétée par le Protocole relatif aux droits des femmes. Ce protocolen'est pas encore été adopté par le Burundi, mais ce dernier l'a uniquement signé voir http://www.africaunion.org/root/au/Documents/Treaties/ treaties_fr.htm) consulté le 30.10.2013.

19 On distingueen plus de la CADHP, la Convention européenne des droits de l'homme, la Convention interaméricaine de droits de l'homme.

20 Voir l'article 2 « Toute personne a droit à la jouissance des droits et libertés reconnus et garantis dans la présente Charte sans distinction aucune, notamment de race, d'ethnie, de couleur, de sexe, de langue, de religion, d'opinion politique ou de toute autre opinion, d'origine nationale ou sociale, de fortune, de naissance ou de toute autre situation. " Voir aussi l'article 3«.Toutes les personnes bénéficient d'une totale égalité devant la loi. Toutes les personnes ont droit à une égale protection de la loi. » Voir enfin l'article 18 «1.La famille est l'élément naturel et la base de la société. Elle doit être protégée par l'Etat qui doit veiller à sa santé physique et morale.

2. L'Etat a l'obligation d'assister la famille dans sa mission de gardienne de la morale et des valeurs traditionnelles reconnues par la Communauté.

3. L'Etat a le devoir de veiller à l'élimination de toute discrimination contre la femme et d'assurer la protection des droits de la femme et de l'enfant tels que stipulés dans les déclarations et conventions internationales.

4. Les personnes âgées ou handicapées ont également droit à des mesures spécifiques de protection en rapport avec leurs besoins physiques ou moraux.».

21 La charte a été ratifiée par le Burundipar le biais du D.L n 1/29 du 28 juillet 1991. 
D'abord, par le biais de l'article $19^{22}$ déjà cité à maintes reprises. Ensuite au travers d'autres dispositions constitutionnelles ${ }^{23}$.

\section{LES REGLES DE DEVOLUTION SUCCESSORALE AU BURUNDIFACE AU PRINCIPED'EGALITE ET DE NON-DISCRIMINATION : UNE CONTRADICTION A PEINE VOILEE}

Dans la première partie de l'article, il a été démontré que le droit burundais reconnaît l'égalité et prohibe la discrimination basée sur le sexe. Paradoxalement, le droit successoral burundais, dominé foncièrement par la coutume,ne reconnaît pas de principe l'égalité entre l'homme et la femme et consacre de ce fait une discrimination basée sur le sexe.

Certes, une évolution vers l'égalité est consacrée de plus en plus par la jurisprudence, mais cette dernière n'arrive pas à être uniforme sur toute l'étendue du territoire. Par conséquent, il s'observe une certaine forme de dualisme jurisprudentiel, l'égalité et la non-discrimination étant acceptées dans certaines parties du territoiremais pasdans d'autres ${ }^{24}$. Même dans les milieux ruraux, cette deuxième partie est consacrée àdémontrer cette réalité et à tenter de trouver une explication.

A. La place de la femme dans la dévolution successorale en droit coutumier pur : une discrimination évidente.

$1^{\circ}$ Principes

En droit coutumier burundais, il était sans contesteet de principe que la femme (fille) faisait l'objet de discrimination en matière de succession. C'est BUKERA J.qui l'a démontré en

22 L'intégralité de l'article est ainsi libellé «Les droits et devoirs proclamés et garantis, entre autres, par la Déclaration universelle des droits de l'homme, les Pactes internationaux relatifs aux droits de l'homme, la Charte africaine des droits de l'homme et des peuples, la Convention sur l'élimination de toutes les formes de discrimination à l'égard des femmes et la Convention relative aux droits de l'enfant font partie intégrante de la Constitution de la République du Burundi. Ces droits fondamentaux ne font l'objet d'aucune restriction ou dérogation, sauf dans certaines circonstances justifiables par l'intérêt général ou la protection d'un droit fondamental. ».

23 Ainsi del'article13 « Tous les burundais sont égaux en mérite et en dignité. Tous les citoyens jouissent des mêmes droits et ont droit à la même protection de la loi. Aucun burundais ne sera exclu de la vie sociale, économique ou politique de la nation du fait de sa race, de sa langue, de sa religion, de son sexe ou de son origine ethnique. Ainsi de l'article 22 «Tous les citoyens sont égaux devant la loi, qui leur assure une protection égale. Nul ne peut être l'objet de discrimination du fait notamment de son origine, de sa race, de son ethnie, de son sexe, de sa couleur, de sa langue, de sa situation sociale, de ses convictions religieuses, philosophiques ou politiques ou du fait d'un handicap physique ou mental ou du fait d'être porteur du VIH/SIDA ou toute autre maladie incurable.».

24 Il y a lieu de signaler que si ce dualisme jurisprudentiel est très marquéparl'opposition ville-campagne,toutes les régions de la campagne ne sont pas logées à la même enseigne. L'évolution vers l'égalité est ainsi à rythme inégale. 
indiquant l'ordre des successibles ${ }^{25}$.La fille ou la femme occupait la 5è position dans l'ordre des successibles.De fait, elleétait exclue dela succession au bénéfice des seuls enfants de sexe masculin. La discrimination basée sur le sexeétait consacrée et cela aussi bien en ce qui est de la succession ab intestat ou de la succession testamentaire ${ }^{26}$.Certes, les femmes ou filles pouvaient jouir de certains droits limités mais ces derniers ne pouvaient en aucun cas être équivalents à ceux reconnus aux hommes ${ }^{27}$.

\section{$2^{\circ}$ Facteurs explicatifs}

Parmi les facteurs expliquant cette inégalité entre l'homme et la femme quant à la succession, on peutsignaler deuxprincipaux : la structure patriarcale de la société burundaise ainsi que lastructure patrilinéaire du Burundi.

a) La structure patriarcale de la sociétéburundaise

Le Burundi, à l'instar d'autres pays dans le monde,était et est toujours dominé par le système patriarcal. ${ }^{2829}$

Abeda Sultana dans son article explique le concept de patriarcat en ces termes :

"The word 'patriarchy' literally means the rule of the father or the 'patriarch', and originally it was used to describe a specific type of 'male-dominated family' - the large household of the patriarch which included women, junior men, children, slaves and domestic servants all under the rule of this dominant male. Now it is used more generally "to refer to male domination, to the power relationships by which men do-

25 Voir BUKERA, J., La dévolution successorale en droit burundais, in RAJB, $\mathrm{n}^{\circ} 20$. Il distingue en ce qui concerne la successiondeux sortes d'héritiers : les héritiers légitimes et les héritiers irréguliers. Parmi les héritiers légitimes, le premier ordre est constitué par les enfants légitimes et leurs descendants de sexe masculin. Le deuxième ordre est constitué par le père et la mère du défunt. Le troisième par les frères du défunt et leurs descendants mâles, le quatrième ordre est constitué par les oncles paternels du défunt et leurs descendants mâles. Le cinquième ordre est constitué par une fille, une sœur, une tante paternelle, une cousine, ou tout autre parent de la ligne paternelle du défunt.

26 Sur la succession testamentaire, voir MABUSHI, C. La succession testamentaire en droit coutumier burundais, in RAJB, $\mathrm{n}^{\circ} 21$.

27 La fille non mariée avait droit à une portion de terre (icibare). La femme mariée avait droit à une portion de la terre représentant les cadeaux qu'elle recevait de ses parents. La femmedivorcée ou veuve avait droit à une part qui lui assurait sa subsistance. Pour plus amples détails, voir BUKURU, S., La problématique de la succession de la fille (femme) au Burundi. Etat de la jurisprudence. Mémoire, U.B, Fac.de droit, 2003.

28 Voir Etude sur les violences faites aux femmesréalisée par la Chaire Unesco, p.64.

29 Pour plus amples details sur le patriarcat, voir Muhammad M. Haj-Yahia, On the Characteristics of Patriarchal Societies, Gender Inequality, and Wife Abuse: The Case of Palestinian Society, Adalah's Newsletter, Volume 20, November 2005. Voir aussi Suranjita Ray, Understanding Patriarchy, Human Rights, Gender \& Environment. 
La problématique de la mise en œuvre du principe d'égalité en droit burundais de la famille. Cas des droits successoraux de la femme.

minate women, and to characterize a system whereby women are kept subordinate in a number of ways" " ${ }^{30}$.

Il s'agitde fait d'un systèmeinstitutionnalisé de domination de la femme par l'homme aussi bien dans la sphère publique que dans celle privée ${ }^{31}$. Par conséquent, la fille ou la femme est dominéeet discriminéedans plusieurs domaines dont ceux relatifs à l'accès à des ressources, plus particulièrement en ce qui concerne l'héritage. Sultana démontre que parmi les exemples illustratifs de cette discrimination consécutive au patriarcat figure l'absence d'héritage et des droits de propriété pour les femmes ${ }^{32}$.

Mais à côté de ce patriarcat, la structurepatrilinéaire du Burundi explique aussi que les femmes soient discriminées en ce qui concerne la succession.

b) La structure patrilinéaire et agnatique de la famille burundaise

Selon la culturetraditionnelle burundaise, c'est leclan qui supplantaitla famille nucléaire. De même,seuls les hommes étaientsusceptibles de faire la continuation du clanou de la lignée paternelle, à l'exception des femmes ou filles.Ces dernières y participaient, mais c'étaituniquement au sein de la famille du mari et non dans la famille d'origine.En effet,elles étaientcensées « aller ailleurs ${ }^{33}$ », dans une autre famille, dans un autre clan. Par conséquent, elles étaient excluesdela succession, de peur que les biens ne sortent de la famille d'origine pour entrer dans la famille desépoux.

De tout ce qui précède, il est sans conteste que la femme et la fille subissait une discrimination.

Mais si cette inégalité oucette discrimination pouvait être toléréeou acceptée à cette époque-là ${ }^{34}$, peut-il continuer à être le cas depuis que le Burundi est partie à différents instruments de protection de droits de l'homme en général, et de protection de droits de la femme en particulier? Peut-il aussi continuer à être le cas depuis que le Burundi s'est doté d'une constitution reconnaissant l'égalité de tous en droits et en dignité?Notre réponse est négative.

Et pourtant, l'analyse faite de la jurisprudence contemporaine montre que l'inégalité et la discrimination n'ont pas du tout disparu. Certesla situation vécue par les femmes et filles aconnu unecertaine évolution. En effet, les juges-une fois appelés à trancher les litiges en matière successorale (qui sont d'ailleurs nombreux dans les tribunaux de résidence)-

30 Abeda Sultana, Patriarchy and Women's Subordination: A Theoretical Analysis, The Arts Faculty Journal, July 2010-June 2011, p.2.

31 Idem, p.3.

32 Idem, p.8.

33 Le dicton burundais « Umukobwa akura iyo agiye » le disait bien.

34 Si on considère que le principe d'égalité n'était pas encoreconsacré en droit burundais. Il faut garder à l'esprit que la plupart des textes fondamentaux des droits de l'homme ont commencé à être ratifiés par le Burundi dans les années 1990avec l'avènement de l'ère démocratique. 
tendent vers de plus en plus d'égalité entre l'homme et la femme. Pour autant, l'unité de la jurisprudence en la matièren'est pas faite.Les jugesdistinguent presque systématiquementselon que les biens à hériter se trouvent en milieu rural ou en milieu urbain.

B. La jurisprudence contemporaine et la place de la femme dans le domaine successoral : un dualisme jurisprudentielconsacré.

a) Observation empirique de la jurisprudence

Face à la coutume purequi discriminait la femme ou la fille en matière successorale,la jurisprudence a fait évoluer leur place ${ }^{35} .{ }^{36}$.Par la suite, toujours en suivant cette évolution,il s'observe un dualisme jurisprudentiel selon qu'on est enmilieu urbainou enmilieu rural.

Si dans le milieu urbain, l'égalité et la non-discrimination sontde plus en plus consacréespar les juges, il n'en va pas de même dans le milieu rural là oùl'inégalité reste une réalité.

Ce dualisme est pourtant inacceptable.

$1^{\circ}$ ) La dévolution successorale de la femmedans le milieu urbain : unereconnaissancejurisprudentielle de l'égalité.

En consultant les différents jugements et arrêts rendus en milieu urbain, à propos des litiges successoraux, il se dégage de plus en plus un fait : les filles et les garçons sont traités sur le même pied d'égalité, les femmes ne sont plus discriminées, du moins les juges n'acceptent pas de couvrir la discrimination basée sur le sexe. ${ }^{37}$

C'est d'une tout autre logique que suivent les juridictions dans le milieu rural.

35 Voir le jugement du tribunal Mwami du Burundi du 9 février 1960, in Revue Juridique du Rwanda et du Burundi, Mai 1963, n³, p.102. Ce jugement reconnaît la possibilité pour les filles, en l'absence de garçons, d'hériter des biens de leur père. Comme on peut s'en rendre compte, ce n'était pas un impératif d'égalité qui a guidé cette solution, puisque ce n'est qu'en absence de garçons qu'elles peuvent hériter. Cependant, ce jugement est important dans la mesure où il bat en brèche la structure patrilinéaire du Burundi. En effet, une fois les filles appelées à la succession et hériter en pleine propriété, à l'exclusion des autres membres de la famille du père, rien n'empêche à leur tour de transmettre cet héritage à leurs enfants, qui sont absolument des étrangers à la famille du père décédé.

36 Voir aussi l'arrêt de la Cour de Cassation du Burundi du 28 octobre 1964. Selon cet arrêt, les filles non mariées et les femmes répudiées par leurs époux doivent être appelés à la succession de leur père. On comprend aussi ici que ce n'est pas pour des considérations d'égalité que les filles étaient admises à la succession, mais plutôt pour des raisons humanitaires. Généralement, elles ne recevaient qu'une portion de la terre, sans équivalent aux parts qui revenaient aux hommes.

37 Voir les différents arrêts jugements commentés par Suzanne BUKURU dans, BUKURU, S.op.citpp.39-44. Ce constat est par ailleurs confirmé dans d'autres travaux de fin d'études déjà faits à la faculté de droit sur le thème de « la jurisprudence des tribunaux de résidence matière successorale » dans presque toutes les provinces du pays. 
$2^{\circ}$ ) La dévolution successorale de la femme dans le milieu rural : une discrimination maintenue au détriment des femmes.

De tous les jugements accessibles sur la succession dans le milieu rural, aucun n'a pu conclure de manière non équivoqueà l'égalité entre les hommes et les femmes. C'est le droit coutumier tel qu'il avait déjà été fixé par la jurisprudence des années $1960^{38}$ qui continue à être appliquémême actuellement. ${ }^{39}$

\section{b) Les facteurs explicatifs de cette dualité jurisprudentielle}

Une telle dualité jurisprudentielle, une telle dispersion de jurisprudence est susceptible de plusieurs explications :

$1^{\circ}$ ) L'absence d'une loi écrite régissant la matière de succession

La matière des successions est, à côté des régimes matrimoniaux et des libéralités, une des matières qui n'est toujours pas régie par une loi écrite ${ }^{40}$. Encore aujourd'hui,pour régler des questions touchant les successions, les juges se réfèrentdans la plupart des cas au droit coutumier ${ }^{41}$.

Et pourtant, un mouvement revendiquant la mise sur pied d'une loi existe depuis longtemps, mais sans succès jusqu'ici. ${ }^{42}$ Les raisons qui expliquent cet état de fait restent à notre

38 Les femmes mariées recueillent une part (qui n'est pas l'équivalent à celui des hommes). Les filles recueillent en propriété, en l'absence des garçons. Les femmes divorcées ou filles célibataires n'ont droit qu'à une part leur permettant de vivre décemment, sans possibilité de la vendre ou de la léguer.

39 Voir les différents arrêts commentés par Suzanne BUKURU in BUKURU, S. op.cit, pp.39-44.

40 Il faut toutefois reconnaitre que d'autres matières touchant le statut personnel et familial sont aujourd'hui réglementés par des textes écrits même si l'évolution du droit coutumier au droit écrit fut particulièrement lente dans le domaine des personnes et de la famille. Cette prudence étaitexpliquée par le souci d'éviter de prendre des mesures impopulaires vis-à-vis des particuliers car on estimait qu'une société est beaucoup plus sensible aux changements qui touchent l'organisation sociale qu'aux institutions politiques (voir le cours d'Aspects de l'Evolution historique du droit burundais, inédit.).

41 Voir supra, le point concernant la dévolution successorale des femmes.

42 En 1975 déjà, dans une de ses résolutions lors d'un congrès national du $1^{\mathrm{er}}$ au 4 septembre tenu à Bujumbura, l'U.F.B (l'Union des Femmes Burundaises) demandait qu'une loi soit promulguée permettant à la femme célibataire ou mariée d'hériter des biens meubles ou immeubles au même titre que les autres enfants. En 2006, grâce à l'activisme de certaines organisations de la société civile, une proposition de loi régissant entre autres les successions a été déposée à l'Assemblée Nationale. Elle n'a toutefois été mise à son ordre du jour jusqu'aujourd'hui. 
sens l'absence de volonté politique des institutions étatiques ${ }^{43}$ accompagnée des résistances culturelles aux changements ainsi quela problématique de l'exiguïté des terres.

Cette absence de loi écrite justifie la dispersion de la jurisprudence en matière de succession. En effet,en l'absence d'une loi écrite ou de disposition législative, les juges ont tendance à appliquer la coutume. Or, cette coutume n'est pas uniforme sur toute l'étendue du territoire. Elle se diversifie selon les régions.C'est ainsi que la jurisprudence en est arrivée à cette conclusion qu'il n'y a pas une même coutumeselon qu'on est en milieu urbain ou en milieu rural, et que même dans ce dernier milieu, l'uniformité de la coutume n'est pas toujours au rendez-vous.

Nous verrons néanmoins plus loin que cette absence de loi écrite ne constitue pas une excuse légitime pour les juges qui n'appliquent pas l'égalité et la non- discrimination en matière successorale.

\section{$2^{\circ}$ ) Le statut particulier du bien foncier en milieu rural}

Comme il a déjà été dit, c'est en milieu ruralque les résistances à l'égalité se remarquent essentiellement. Et c'est surtoutla propriété foncière qui constituele point d'achoppement. Et pour cause. La terre constituaitdans l'imaginaire collectif burundais unbien siprécieuxqu'elle devait demeurerdans le giron familialou clanique.S'il estajoutéà cela la structure patrilinéaire de la société burundaise, la structure agnatique de la famille burundaiseainsi que la virilocalité du mariage, les femmes ne pouvaient pas jouir des mêmes droitsque les hommes sur la propriété foncière rurale.

Par contre, en milieu urbain, il est admis que les biens à hériter sont dans la plupart des cas des biens qui ne sont pas la terre, et donc ne posent pas de problèmes à partager équitablement : argent, maison,...

Nous montreronscependantque ces arguments ne sont pas du tout convaincants.

\section{LE PRINCIPE D'EGALITE FACE A DES PRATIQUES COUTUMIERES CONTRAIRES : A QUOI REVIENT LA PREEMINENCE?}

Il a été démontré dans la première partie de cet article que le principe général d'égalité et dela non-discrimination ont, outre une valeur conventionnelle, mais aussi une valeur constitutionnelle. Il a été indiqué en outre dans la deuxième partie que lespratiques coutumières suivies en ce qui concerne la succession ne sont pas toujours en harmonie avec ces principes.En effet, il a été révéléque les juges saucissonnent leur jurisprudence selon que les femmes veulent hériter les biens situés en milieu rural ou en milieu urbain. Mais, ce dua-

43 Signalons que le Burundi a ratifié entre autres la Convention sur l'Elimination de toutes formes de discriminations à l'égard des femmes et que de ce fait il s'est engagé à « Prendre toutes les mesures appropriées, y compris des dispositions législatives, pour modifier ou abroger toute loi, disposition réglementaire, coutume ou pratique qui constitue une discrimination à l'égard des femmes; "(voir Supra sur le point relatif à la Convention). 
La problématique de la mise en œuvre du principe d'égalité en droit burundais de la famille. Cas des droits successoraux de la femme.

lisme jurisprudentiel est-il en harmonie avec la constitution? Quelle doit être la réaction des juges si un litige leurest soumis ayant trait à la revendication de l'égalité entre l'homme et la femme en matière successorale?

\section{A. LE DUALISME JURISPRUDENTIEL EN MATIERE SUCCESSORALE : LES RAISONS D'UNE NON-PERTINENCE}

Le dualisme jurisprudentiel qui consiste à admettre l'égalité entre les hommes et les femmes dans le milieu urbain et la rejeter dans le milieu rural n'est pas à notre sens pertinent. Au moins trois raisons nous poussentà défendre ce point de vue :

1. La Constitution est une loi suprême et applicable sur toute l'étendue du territoire de la République.

La constitution de la République du Burundi ne démontre pas elle-même la hiérarchie des normes juridiques en droit burundais.Elle ne montre même paselle-même la place qu'occupe la coutume parmi les règles juridiques ${ }^{44}$. Elle donne toutefois sapropre place dans cette hiérarchie. Selon l'article 48 de la constitution de la République du Burundi « (....) La Constitution est la loi suprême. " Or, il a déjà été démontré que la Constitution a non seulement intégré en son sein la plupart des textes internationaux prônant l'égalité ou prohibant la discrimination faite aux femmes, mais aussi prône elle-même l'égalité en droit et en dignité.

Par ailleurs, la constitution, loi suprême du pays, ne prévoit pas une différenciation dans sa mise en œuvre selon le territoire du pays. Elle doit s'appliquer partout. Elle ne devrait pas s'appliquer à plusieurs vitesses.Une pratique coutumière, quelqu'en soit le lieu où elle existe en contradiction avec la constitution est en principe irrelevante.

2. Le caractère patrilinéaire de la société burundaiseest de moins en moins pertinent

Il a déjà été souligné que le caractère patrilinéaire de la société burundaise militait pour l'exclusion de la fille à l'héritage ou pour la reconnaissance du droit de propriété sur la terre. En effet, selon ce système, seuls les garçons perpétuent la famille, et il ne fallait pas que plusieurs famillesse partagent une même terre. Or, avecla jurisprudence reconnaissant le droit successoral des filles et femmes, en cas d'absence de garçons, et cela à l'exclusion des autres membres de la famille du «de cujus », c'est le système patrilinéaire qui est botté

44 Sur la place de la coutume dans la hiérarchie des normes juridiques en droit burundais, il faut se référer à l'ordonnance de l'administrateur général du Congodu 14 mai 1886. Cette ordonnance rendue exécutoire au Burundi par O.R.U. n 11/82 du 21 juin 1949, dispose que « Quant la matière n'est pas prévue par un décret, un arrêté ou une ordonnance déjà promulguée, les contestations qui sont de la compétence des tribunaux du Congo seront jugées d'après les coutumes locales, les principes généraux du droit et l'équité »(Voir Codes et Lois du Burundi (31 décembre 2006), T1, p. 202. 
en touche. En effet, en pareille hypothèse,et selon les propos de Gervais Gatunange, « ces biens familiaux dont héritent les filles passeront finalement à leurs enfants qui, par définition n'appartiennent pas au même lignage. ${ }^{45}$ ». Par conséquent, des membres de familles différentes vivent sur une même terre et pareille positon est généralement acceptée et est même appliquée de manière constante par les juridictions qui admettent aux fillesen cette circonstancetoutes les prérogatives du droit de propriété.

\section{Tous les attributs de la propriété foncière en milieu rural sont de plus en plus reconnus}

Il est aujourd'hui admis que la terre en milieu rural n'est pas du tout un bien appartenant au clan. Au contraire, il devient de plus en plus un biensur lequel il peut être exercé des droits privatifs en accord avec les attributs du droit de propriété. Plusieurs arguments le démontrent.

D'abord, lecode foncier du Burundi qui fixe les règles qui déterminent les droits fonciers reconnus ou pouvant être reconnus sur l'ensemble des terres situées sur le territoire national ${ }^{46}$,reconnait que toute personne physique ou morale peut jouir, sans discrimination aucune, de tous les droitsqu'il définit ${ }^{47}$. Et parmi les droits reconnus figure bien entendu le droit de propriété foncière ${ }^{48}$ avec tous ses attributs.

Ensuite, selon le code des personnes et de la famille-texte applicable sur tout le territoire national- chaque époux a le droit-bien sûr avec le consentement de l'autre épouxd'aliéner ou grever des droits réels les immeubles ou les exploitations dépendant de la communauté conjugale. Et parmi les biens réputés dépendants de la communauté conjugale figurent entre autres les fonds de terre acquis par dévolution successorale ${ }^{49} .{ }^{50}$

Enfin, la pratique va dans le sens de ces dispositions théoriques. Des terres en milieu rural font l'objet de transaction ${ }^{51}$.

Toutes cesraisons militent pour la reconnaissance aux femmes fussent-elles celles du milieu rural,des droits égaux à ceux reconnus aux hommes en matière successorale. Faire le contraire serait perpétuer une discrimination inacceptable.

45 GATUNANGE, G., Femmes et mariage en droit traditionnel burundais : l'émancipation de la femme burundaise par l'approfondissement des valeurs de civilisation nationale, thèse, UCL, 1982, p.364.

46 Voir loi n $1 / 013$ du 9 août 2011 portant révision du code foncier du Burundi, article 1.

47 Idem, art. 12.

48 Idem, art.16.

49 Voir Décret-loi n 1/024 du 28 avril 1993 portant réforme du code des personnes et de la famille, article 126.

50 Or selon la coutume, ce sont ces fonds de terre acquis par dévolution successorale qui ne devraient en principe pas quitter le giron familial.

51 L'auteur de cet article reconnaît que son parenta a acheté un terrain en milieu rural dont les anciens propriétaires ne partagent pas du tout la famille, voire même le clan avec lui. Cela vient d'ailleurs renforcer la perte de la pertinence de l'argument selon lequeldeux ou plusieurs familles ou clansne peuvent pascohabiter sur un même terrain. 
Et si ce dualisme jurisprudentiel est inacceptable, comment les juges devraient-ils trancher afin d'uniformiser leur jurisprudence? Plusieurs solutions s'imposent à euxafin de pouvoir réaliser l'égalité et lutter contre la discrimination faites aux femmes.

\section{B. ESSAI DE SOLUTIONS OFFERTES AUX JUGES AFIN D'ASSURER L'UNITE JURISPRUDENTIELLE PRONANT L'EGALITE.}

Face à cette problématique liée à la discrimination faite aux femmes et aux filles en matière de succession, plusieurs solutions pourraient être adoptées par les juges. Au lieu de continuer à appliquer une coutume contraire aux principes reconnus par la constitution et les conventions internationales, ils pourraient adopter des solutions qui vont dans le sens du respect de ces principes. Certes, leur tâche aurait été facile s'il y avait une loi écrite et claire. Mais l'absence d'une telle loi n'empêcherait pas de s'inspirer d'autres sources de droit.

\section{Donnerprimauté à la Constitution}

Il a déjà été démontré que la constitution de la République du Burundi est une norme suprême. Les juges devraient alors la faire primer sur toute norme inférieure, qu'il s'agisse d'une loi ou d'une norme coutumière.En effet, il est évident qu'au Burundi, une loi qui n'est pas en conformité avec la constitution serait nulle (voir art 48 in fine de la constitution). Il en estsans doute de même d'une norme coutumière, quitrouve à s'appliquer en l'absence d'une loi écrite. Or, cette constitution, en reconnaissant les principes d'égalité et de non-discrimination devrait recevoir une primauté par rapport à une norme coutumière contraire.

Il s'agit par ailleurs d'une obligation pour les juges de faire respecter la Constitution ainsi queles droits fondamentaux qu'elle consacre ${ }^{52}$.

Signalons aussi que l'article 209 de la constitution dispose que «dans l'exercice de ses fonctions, le juge n'est soumis qu'à la Constitution et à la loi. ».

Ainsi donc, en donnant une primauté à la constitution, et donc en refusant de consacrer une quelconque discrimination à l'endroit des femmes en matière successorale, le juge n'aura commis aucune erreur. Au contraire. Il aura bien rempli son rôle de gardien des libertés fondamentales. C'est ce qu'affirme Maurice Kamto lorsqu'il écrit que

« en raison de la constitutionnalisation des droits, leur garantie juridictionnelle [...] se fait, d'une part, par le biais du contrôle de la constitutionnalité des lois et d'autre part, par le biais du contentieux des droits et libertés. $\|^{53}$

52 Selon l'article 48 de la constitution «Les droits fondamentaux doivent être respectés dans l'ensemble de l'ordre juridique, administratif et institutionnel. La Constitution est la loi suprême. Le pouvoir législatif, l'exécutif et le judiciaire doivent la faire respecter (....)».

53 Maurice Kamto, Charte africaine, instruments internationaux de protection des droits de l'homme,constitutions nationales :articulations respectives; Jean-François Flauss et Elisabeth Lam- 
2. Appliquer directement les instruments internationaux

Outre que pour consacrer l'égalité et donc lutter contre la discrimination faite aux femmes en matière successorale le juge peut donner primautéà la constitution, ce dernier peut aussi opter pour l'applicationdirecte des instruments internationaux pertinents. En effet, le Burundi est un Etat moniste mais aussi les instruments internationaux ont primauté surles pratiques coutumières.

\section{a. Le Burundi est un Etat moniste}

Les auteurs classiques distinguent deux systèmes de domestication du droit international en droit interne : le monisme et le dualisme ${ }^{54}$.

D'après le monisme, le droit international et le droit interne forment un seul système juridique et le traité international est incorporé directement dans le droit interne dès sa ratification.Par conséquent, juge national pourra appliquerdirectementla norme internationale dès cette ratification.

En revanche, le dualisme considère que les deux systèmes sont séparés et doncqu'un traité international ne peut produire d'effets que s'il est transformé en règle de droit interne.

Il appartient à chaque Etat dedéterminerdans sa constitution lequel parmi ces systèmes est le plus approprié.

A cet égard, le Burundi reconnaît lesystème moniste. En effet, aux termes de l'article 292 de la constitution du 18 mars 2005,

"Les traités ne prennent effet qu'après avoir été régulièrement ratifiés et sous réserve de leur application par l'autre partie, pour les traités bilatéraux, et de la réalisation des conditions de mise en vigueur prévues par eux, pour les traités multilaté$\operatorname{raux} \gg .55$

bert-Abdelgawad (dir.),L'application nationale de la charte africaine des droits de l'homme et des peuples,Bruylant, Bruxelles, 2004 pp 37-38 cité par Nyaluma Mulagano Arnold, Le juge congolais et le principe d'égalité : sort des droits de la femme dans la jurisprudence du tribunal de grande instance de Bukavu, in Librairie africaine d'études juridiques, Vol,3, p.99.

54 Pour plus de détails, voir F.X Bangamwabo, The implementation of international and regional human rights instruments in the Namibian legal framework, Yuval Shany, How supreme is the supreme law of the land? comparative analysis of the influence of international human rights treaties upon the interpretation of constitutional texts by domestic courts(2006), Brook. J. Int'l L., Melissa A. WATERS, Creeping Monism: The Judicial Trend Toward Interpretive Incorporation Of Human Rights Treaties, Columbia Law Review. 
La ratification relève soit du Président de la République ${ }^{56}$ soit du Parlement ${ }^{57}$. S'agissant de cette dernière hypothèse, il est possible de croire que pour certains traités, du fait qu'ils nécessitent une intervention législative, le Burundileur réserve un système dualiste.Mais il n'en est rien.Il ne s'agit que d'une atténuation du monisme qui n'enlève en rien le caractère moniste du système burundais ainsi que le fait savoir Nestor Nkurunziza enparaphrasant Stef Vandenginste en ces termes :

"Vandenginste fait remarquer que la nature moniste du système burundais se trouvetempéré du fait que la ratification de certaines catégories de traités relève de la compétence du législateur. Ce tempérament n'entraîne pas pour autant une perte du caractère moniste puisque, comme cet auteur le note, la ratification, même par le parlement, consiste le plus souvent, en un article uniqueconsacrant ainsi l'entrée de la Convention dans l'ordre interne. Or, dans l'hypothèse d'un système dualiste, l'acte de "domestication" du traité consisterait en l'adoption d'une loi lloi de réception) par le législateur national reprenant intégralement le contenu du traité, disposition par disposition le cas échéantpuisque, dans une conception dualiste, le droit interne et le droit international constituent deux blocs séparés. Dans un système moniste, par contre, les deux sources du droit appartiennent à la même catégorie. $\|^{58}$

Par ailleurs, le caractère moniste du Burundia été reconnu à de nombreusesautres occasions ${ }^{59}$

Ainsi donc, les juges pourraient invoquer directement les textes internationaux pertinents consacrant l'égalité et prohibant la discrimination.La jurisprudence burundaise ne manque d'ailleurs pas d'exemples dans ce sens. En effet,aussi bien la Cour constitution-

56 L'article 289 de la constitution dispose que «Le Président de la République a la haute direction des négociations internationales. Il signe et ratifie les traités et accords internationaux ». (C'est nous qui soulignons.).

57 L'article 290 dispose que "Les traités de paix et les traités de commerce, les traités relatifs à l'organisation internationale, les traités qui engagent les finances de l'Etat, ceux qui modifient les dispositions de nature législative ainsi que ceux qui sont relatifs à l'état des personnes ne peuvent être ratifiés qu'en vertu d'une loi. ».

58 Nestor Nkurunziza,Les défis à la saisine du mécanisme régional de protection des droits de l'homme par les victimes présumées des violations des droits de l'homme au Burundi, T.F.E, DESS, Bujumbura, décembre 2013, p. 36.

59 Idem, pp 35-37. 
nelle ${ }^{60}$ que la Chambre de Cassation ${ }^{61}$ ontdéjà appliqué directement les instruments internationaux.

b. Les instruments internationaux priment sur les lois et sur les pratiques coutumières

Après avoir discuté la problématique de l'applicabilité directe des instruments internationaux, il se pose celle du rang de la norme internationale dans l'ordre interne.

Mais le débat existe seulement à propos du rang de la norme internationale par rapport à la Constitution. Pour les uns, la norme internationale a un rang supra-constitutionnel ${ }^{62}$ tandis que pour d'autres, la norme internationale a un rangsoit constitutionnelle soitinfraconstitutionnel. ${ }^{63}$

S'agissant du rang de la norme internationale par rapport aux sources infra-constitutionnelles, il semble qu'il y aitconsensus : la première supplante les deuxièmes. Certes, cette position est clairement affirmée s'agissant du rang de la norme internationale par rapport aux lois et règlements ${ }^{64}$. Mais qu'en est-ildu rang de la norme internationale par rapport à une normecoutumière? Notre position est que si la norme internationale supplante les lois et les règlements, elle supplanteaussi une norme coutumière, étant donné que celle-ci ne s'applique qu'en l' absence de texte écrit régissant la matière concernée.

En conclusion, tout ce débat concernant l'applicabilité directe ainsi que le rang de la norme internationale en droit burundaisa été tranché par le Constituant burundais en son article 19 sus vu qui a donné la valeur constitutionnelle aux droits et devoirs garantis par les instruments internationaux relatifs aux droits de l'homme. Ainsi donc, le juge en écartant la

60 Voir arrêt RCCB 8 «Attendu en effet que l'article 10 de la Déclaration Universelle des Droits de l'Homme et l'article 14 alinéa premier du Pacte international relatif aux droits civils et politiques, applicables par l'effet de l'article 10 de la Constitution consacrent... ».

61 Cour Suprême, chambre de cassation, Maniraho Ange c. Ministère public, RPC 1301, Arrêt du 28 juin 2001, « que le Pacte International Relatif aux Droits Civils et Politiques qui dispose qu'on ne peut pas être témoin dans son propre procès pouvait s'appliquer dans le cas de Odette Bunama (coauteur) ", publié dans Avocats Sans Frontières, Burundi. Recueil de Jurisprudence. Matières pénales, T.I, 2002, Bujumbura, p.56.

62 L'Etat face aux droits sociaux de ses citoyens: cas du droit à la santé au Burundi.. Voir aussi dans ce sens,MANIRAKIZA, E.,Application du droit international au Burundi, p.9 (inédit.).

63 Voir dans ce sens NKURUNZIZA,N.,op.cit,pp.39-41.

64 La position officielle du Burundi a déjà été démontrée en ces termes : « Le Pacte et la Constitution priment naturellementsur le Code Pénal et le Code de Procédure Pénale. Si ces derniers n'étaient pas en conformité avec le Pacte ou la Constitution, ils étaient automatiquement amendés; de même, toute décision en violation de la Constitution ou des droits fondamentaux était susceptible d'annulation par la Division d'Appel ». Voir NKURUNZIZA, N., op.cit, p.39.(c'est nous qui soulignons). Cette position officielle a été réitérée à une autre occasion en ces termes « L'Etat du Burundi reconnaît que les normes internationales auxquelles il a souscrit sont hiérarchiquement supérieures à ses lois et règlementsRapport initial du Burundi. (voir NKURUNZIZA, N. op.cit, p. 39 , note 106.). 
La problématique de la mise en œuvre du principe d'égalité en droit burundais de la famille. Cas des droits successoraux de la femme.

coutume par l'application directe des instruments protégeant les droits fondamentaux ne ferait que respecter la Constitution, ce qui est d'ailleurs son devoir suprême.

\section{CONCLUSION}

Cet article avait pour objectif de discuter la problématique de la mise en œuvre du principe d'égalité en matière de droits successoraux de la femme burundaise.

Il a été démontré que bien que le Burundi soit partie à plusieurs instruments internationaux relatifs aux droits de l'homme et consacrant l'égalitéet qu'il se soitmême doté d'une Constitution progressiste en matière d'égalité, cette égalité est loin d'être effectivement mise en œuvre s'agissant des droits successoraux des femmes. Certes, il s'observe une certaine évolution pour les femmes vivant dans les milieux urbains alors que les femmes vivant dans les milieux ruraux continuent à subir les affres de la discrimination.Plusieurs facteurs qui constituent autant d'obstaclesexpliquent cet état de fait : l'absence d'une loi écrite sur les successions, lesstructures patriarcale, patrilinéaire et agnatique de la famille traditionnelle burundaise, les résistances aux changements,... Il s'agit toutefois des facteurs qui à notre sens peuvent être surmontés par l'œuvre des juges.

C'est ainsi que nous avons suggéré que les juges-une fois appelés à trancher un litige successoral impliquant les hommes et les femmes-écartent toute pratique qui serait en contradiction avec les principes d'égalité et de non-discrimination. Il s'agit en effet des principes constitutionnels qui doivent s'appliquer sur toute l'étendue du territoire de la République et les juges sont tenus de faire respecter la Constitution, loi suprême du pays.

\section{BIBLIOGRAPHIE}

I. Textes internationaux et régionaux

1. Déclaration Universelle des droits de l'homme et des peuples

2. Pacte International Relatif aux Droits Civils et Politiques. InRecueil des Traités, .

3. Pacte International Relatif aux Droits Economiques, Sociaux et Culturels. In Recueil des Traités.

4. Convention sur l'élimination de toutes les formes de discriminations faites aux femmes in Nations Unies, Recueil des Traités, vol.1249, p.13

5. Charte Africaine des Droits de l'Homme et des Peuples, . In21 I.L.M. 58.

II. Textes constitutionnel et législatifs internes

1. Loi $\mathrm{N}^{\circ} 1 / 010 \mathrm{du} 18$ mars 2005 portant promulgation de la Constitution de la République du Burundi

2. Loi $n^{\circ} 1 / 013$ du 9 août 2011 portant révision du code foncier du Burundi

3. Décret-loi $n^{\circ} 1 / 024$ du 28 avril 1993 portant réforme du code des personnes et de la famille 
III. Ouvrages, Articles, Thèse etMémoire

1. Bukera, J., La dévolution successorale en droit burundais, in RAJB, $n^{\circ} 20$

2. Catarina K. and Martin S (eds.), Combating Discrimination Based on Sex and Gender,International Protection of Human Rights(Turku :Institute for Human Rights, Abo Akademy University,2009)

3. Gatunange, G., Femmes et mariage en droit traditionnel burundais : l'émancipation de la femme burundaise par l'approfondissement des valeurs de civilisation nationale, thèse, UCL, 1982.

4. Hannum, H.The Status of the Universal Declaration of Human Rights in National and International Law, Georgia Journal of International and Comparative Law, Vol 25,1995-1996.

5. Kamto,M.,Charte africaine, instruments internationaux de protection des droits de l'homme, constitutions nationales :articulations respectives in Jean-François Flauss et Elisabeth Lambert-Abdelgawad (dir.),L'application nationale de la charte africaine des droits de l'homme et des peuples,Bruylant, Bruxelles, 2004.

6. Mabushi, C., La succession testamentaire en droit coutumier burundais, in RAJB, $\mathrm{n}^{\circ}$ 21.

7. Manirakiza, E.,Application du droit international au Burundi,(inédit)

8. Manisuli S., (2007). Culture and the Human Rights of Women in Africa: Between Light and Shadow. Journal of African Law, 51, $\mathrm{n}^{\circ} 1$,

9. Manisuli, S., Women's Rights To Equality And Non-Discrimination: Discriminatory Family Legislation In Uganda And The Role Of Uganda's Constitutional Court. International Journal of Law, Policy and the Family21 (2007),

10. Muhammad M., On the Characteristics of Patriarchal Societies, Gender Inequality, and Wife Abuse: The Case of Palestinian Society, Adalah's Newsletter, Volume 20, November 2005.

11. Nkurunziza,N., Les défis à la saisine du mécanisme régional de protection des droits de l'homme par les victimes présumées des violations des droits de l'homme au Burundi.T.F.E, DESS, Bujumbura, décembre 2013.

12. L'Etat face aux droits sociaux de ses citoyens: cas du droit à la santé au Burundi.

13. Nyaluma Mulagano A, Le juge congolais et le principe d'égalité : sort des droits de la femme dans la jurisprudence du tribunal de grande instance de Bukavu, in Librairie africaine d'études juridiques, Vol, 3.

14. Sultana, A., Patriarchy and Women's Subordination: A Theoretical Analysis, The Arts Faculty Journal, July 2010-June 2011.

15. Vandenginste, S. (2011), Stones Left Unturned: Law as a Source and Instrument of Transitional Justice in Burundi. Antwerp, Intersentia

16. Waters, M.A.,Creeping Monism: The Judicial Trend Toward Interpretive Incorporation Of Human Rights Treaties, Columbia Law Review(2007). 
La problématique de la mise en œuvre du principe d'égalité en droit burundais de la famille. Cas des droits successoraux de la femme.

17. Yuval S., How supreme is the supreme law of the land? comparative analysis of the influence of international human rights treaties upon the interpretation of constitutional texts by domestic courts(2006), Brook. J. Int'1 L., 